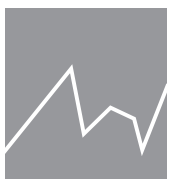

Asociación Cuadernos de economía

\section{Cuadernos de economía}

www.cude.es

ARTÍCULO

\title{
Estrategias del marketing portuario: El caso español
}

\author{
María Jesús Freire Seoane ${ }^{a}$, Carlos Pais Montes ${ }^{b}$ y Fernando González Laxe
}

\author{
a Profesora Emérita de la Universidad de La Coruña \\ b Doctor en Ciencias Económicas por la Universidad de La Coruña \\ c Catedrático de Economía Aplicada de la Universidad de La Coruña
}

\section{JEL CODES \\ R4; \\ M3 \\ KEYWORDS: \\ Ports marketing; Marketing strategies; Connectivity; Analysis of the principal components}

\begin{abstract}
Traditional ports have modified their patterns of behaviour, and currently they are working on strengthening their marketing strategies aiming to address a global market, where increasing competition, demand for new infrastructures as well as easiness to access and to operate are the new norms. This article sets out the news ways and processes to deal with the tasks related to port corporate governance as regards to the promotion of their marketing. The article analyses the quantitative structures in the specific case of the Spanish Ports, from which two strategic lines are obtained: marketing focused on increasing cargo volumes, and marketing focused on increasing connectivity. Finally, the article also quantifies the results of the efforts by the 28 Port Authorities on the marketing field, and presents their positioning using an analysis of the principal components of these indices.
\end{abstract}

\section{CÓDIGOS JEL}

R4;

M3

\section{PALABRAS CLAVE:}

Marketing portuario; Estrategias de marketing; Conectividad; Análisis de componentes principales

\begin{abstract}
Resumen: Los puertos tradicionales han modificado sus hábitos y sus comportamientos y, actualmente, tratan de potenciar sus estrategias de marketing en un mercado global donde existe mayor competencia, y donde cada vez se exigen nuevas infraestructuras y mayores facilidades para operar. En este artículo se describen las nuevas vías a través de las cuales se realizan las tareas de gobernanza portuaria desde el punto de vista de la promoción del marketing portuario. Se analiza la estructura cuantitativa del caso de los puertos españoles obteniéndose dos líneas estratégicas: el marketing orientado hacia el aumento del volumen de carga, y el orientado hacia el aumento de la conectividad. También, se cuantifican las actuaciones de las 28 Autoridades Portuarias en torno a su apuesta en el campo del marketing, y se presentan sus posicionamientos aplicando un análisis de componentes principales a estos índices.
\end{abstract}

a,b,c Correo electrónico: maje@udc.es; laxe@udc.es; carlos.pais.montes@udc.es 


\section{Introducción}

Hasta hace poco tiempo la mayoría de los trabajos y libros publicados sobre marketing territorial o portuario estaban dirigidos hacia la comunicación empresarial o bien versaban sobre los destinos turísticos. Esta inclinación ha ido evolucionado a lo largo de los últimos años, adaptándose tanto a las nuevas necesidades sociales como a los nuevos objetivos empresariales (Kotler, 2000; Kotler y Getner, 2002). De esta forma, el marketing se orientó, en primer término, hacia la producción, de tal forma que se asociaba a las tendencias por las que las empresas definían sus objetivos en términos de volumen o de fabricación en gran escala. En una segunda fase, el marketing se centró en el producto, tanto en sus rasgos de calidad como en su propia diferenciación respecto a los demás. Más tarde, en una tercera fase, el marketing se orientó hacia las ventas, pero sin preocuparse de su relación con los clientes. En la cuarta fase, las preferencias del marketing se centraron en los consumidores, buscando cubrir sus necesidades y ofreciendo sus productos frente a una demanda más exigente y particularizada. $Y$, finalmente, en la quinta fase, predominan la orientación hacia lo social, o sea en función de las necesidades y demandas de la sociedad, y cumpliendo los criterios de la responsabilidad social corporativa.

En la actualidad, el marketing se acerca a las necesidades de gestión y comunicación de las instituciones; ya que un destino o un lugar geográfico se convierten en un producto que se puede comercializar. En base a estos criterios, los entes institucionales pueden llegar a crear sus propias marcas gestionadas de igual forma que otros productos de consumo y de servicios (Anholt, 2002; Caldwell y Freire, 2004). No cabe duda que esta apuesta plantea ciertos problemas en la medida que se trata de procesos difíciles para crear tanto una marca como una imagen; ya sea para un territorio/lugar/institución; ya sea para subrayar una estrategia o un posicionamiento único y diferenciado en un mercado abierto y competitivo.

Este trabajo se centra en evaluar las diferentes opciones desplegadas a la luz de la articulación de propuestas, tanto ofensivas como defensivas que, siendo reflejadas en los medios de comunicación, generalmente, consultados por los stakeholders, muestran las distintas concepciones del posicionamiento empresarial e institucional. Nuestro objetivo es revisar las distintas opciones que desde el campo del marketing se han formulado en el área del sector marítimo-portuario. Los recientes cambios y transformaciones derivadas de las respuestas a la crisis económica, iniciada en 2008, han alentado la proliferación de nuevos posicionamientos institucionales, que han motivado profundos cambios en las dinámicas de alianzas empresariales y han generado nuevas uniones en el campo de la logística y la intermodalidad.

En la investigación hemos aceptado aquellas teorías del branding territorial y empresarial basadas en la competencia y competitividad, para poder enfatizar las estrategias de diferenciación en lo que atañe al posicionamiento empresarial y territorial, siguiendo la tesis de Nandas (2005), por lo que es necesario crear una imagen favorable para poder ascender a mayores oportunidades de mercado, a través del establecimiento de relaciones positivas, aceptando que el territorio y la empresa pueden llegar a ser más atractivas respecto a las demás en un mercado global. De ahí que en el caso marítimo-portuario sea necesario yuxtaponer dos componentes: uno de carácter cognitivo (atributos físicos y tangibles de un destino); y otro, el derivado de una dinámica más competitiva (conjunto de valores con los que se asocia).

Por ello, en el caso de un puerto es preciso añadir aquellos componentes centrados en la inserción geográfica y en la conectividad. En consecuencia, sus dinámicas de creación de marca y de la puesta en marcha de una planificación estratégica y de comunicación, exigen tanto la imbricación de los diferentes agentes, como la participación activa y la responsabilidad en cada uno de los procesos.

En este trabajo, se aborda, en un primer epígrafe una introducción a las distintas opciones que desde el marketing se han formulado en el área del sector marítimo-portuario. En el segundo epígrafe, se hace una revisión de la evolución experimentada por el marketing portuario. En el tercero, se revisa el desarrollo del marketing portuario y los escenarios estratégicos a largo plazo. El cuarto epígrafe, se centra en las orientaciones de mercado de la industria portuaria. En el quinto epígrafe, se hace mención al contexto español en donde se cuantifican las actuaciones de las 28 Autoridades Portuarias en torno a su apuesta en el campo del marketing, y se presentan sus posicionamientos aplicando un análisis de componentes principales a los índices de cada Autoridad Portuaria. Y, finalmente, se exponen las conclusiones más significativas.

\section{Antecedentes}

Branch (1998) ha investigado sobre el marketing de las empresas de navegación; en donde subrayaba cuatro etapas del ciclo de marketing: análisis, planeamiento, complementariedad y control. Dichas etapas identificaban importantes diferencias conceptuales y subrayaba: las variables económicas; las cuestiones relativas a la gobernanza; la rivalidad y la competencia; las cuestiones culturales y demográficas; los aspectos tecnológicos; las restricciones éticas y legales; las variables ecológicas y aquellas situaciones con recursos limitados; la estructura y las cuotas de mercados internacionales; y, finalmente, la cultura de empresa.

Significa, por lo tanto, que con el actual desarrollo de la logística portuaria, una nueva dimensión se añade a la gestión portuaria. Emerge como un criterio relevante el mejor uso posible del tiempo disponible, tanto en los puertos como en las terminales; buscando optimizar el paso por las terminales con la utilización de los servicios que generan mayor valor añadido. Es decir, las Autoridades Portuarias buscan reforzar su imagen; y el nuevo papel de los puertos se encamina a desempeñar un rol muy importante en el comercio y en el desarrollo económico de un país, enfatizando los aspectos de seguridad, fiabilidad y rapidez, así como los relativos a los procedimientos administrativos -facilidad burocrática y evitación de duplicidades- (Notteboom y Wilkenmans, 2002; Robinson, 2002).

Plomaritou (2006), por su parte, explicita cinco fases en lo que hace referencia a la implantación del marketing en las compañías navieras: en primer lugar, la diagnosis; en 
segundo lugar, la planificación; en tercer lugar, la organización; en cuarto lugar, la implementación; y, por último, el control. La primera fase de diagnosis, incluye a su vez a la organización del marketing en lo que se refiere a la información; la fase de análisis del marketing, haciendo mención a las amenazas/oportunidades; la fase de la segmentación de mercados (tipos de buques, rutas, duración del charteo,....); la fase de evaluación de los mercados (tamaño y desarrollo, atractividad de los segmentos); y la fase de elección (nivel de concentración, especialización, servicios y cobertura).

En la teoría moderna de los puertos se insiste en la relevancia de las estrategias de marketing (Cheon, 2007), incluyendo en ellas la comunicación y la imagen para la captura de nuevas líneas y cargas. Tesis que son suscritas en los trabajos de Cahoon (2007), que incluyen la necesidad de definir una estrategia de posicionamiento internacional. Dicha inclusión resulta cada día más imprescindible, hasta el punto de que Notteboom (2011) incluye entre los factores de éxito tanto la calidad de los servicios del puerto como la reputación y el trabajo desarrollado en el ámbito del marketing.

Van der Lugth y de Langen (2007) insisten en el papel desarrollado por aquellas Autoridades Portuarias que recomendaban una mayor integración con su hinterland próximo y asociado; promoviendo la necesaria integración entre las diversas organizaciones, identificando, en consecuencia, aquellas demandas comunes de clientes y usuarios del puerto, buscando solventar y solucionar las dificultades encontradas y existentes en la comunidad local ante el impacto de las actividades portuarias. Por eso, poseen una gran relevancia aquellas actuaciones que poseen como marco de referencia la satisfacción de los clientes, orientando sus actuaciones y sus estrategias de comunicación e imagen en esa línea de conducta (Calderinhina et al., 2013).

En el campo concreto del marketing portuario, destacan las aportaciones de Pando et al. (2005) que orientan sus investigaciones en definir aquellas estrategias de comunicación e instrumentos de marketing que contribuyen a aumentar el desempeño; los estudios de Tongzon y Sawant (2007) analizan el marketing en función de las características del puerto y de las terminales, de cara a evaluar el desempeño del puerto como un resultado de la selección de los armadores. Pardali y Kounoupas (2007) evalúan la oferta del puerto como un instrumento del marketing, escudriñando los diversos factores instrumentales referidos al precio, al producto y a la comunicación; aspectos todos ellos que influyen y condicionan el desempeño portuario; lo que llevó a Caldeirinha (2014) a formular la necesidad de implementar un "port marketing theory".

En la literatura académica existen muchos factores que determinan el desempeño portuario; pero no abundan aquellos que, de forma sistemática, se basen en determinar los instrumentos de marketing. De esta forma, los estudios de los puertos se clasifican en cinco grandes tipos (Chang y Lee, 2008): a) los factores relacionados con la selección de puertos; b) los que inciden sobre las políticas de competitividad; c) los que enfatizan sobre la gobernanza, propiedad y privatizaciones; d) los que analizan las medidas de eficiencia, desempeño y productividad; e) finalmente, los que evalúan las políticas de cooperación, alianzas y adquisiciones. Otros autores, como Pallis et al. (2011), enfatizan sobre otros cinco aspectos: a) el estudio de las terminales, puertos y cadenas logísticas; b) la gobernanza y el planeamiento; c) la política portuaria y las regulaciones; d) la competitividad y la competencia; e) el análisis espacial.

Juang y Roe (2009) verifican tres factores principales sobre las actividades de los puertos y su correspondencia con su imagen: a) los servicios que ofrecen y que prestan; b) las bases de su marketing; y c) la logística disponible. En el primero de los factores, las variables principales hacen referencia al precio a pagar por los mencionados servicios; en el segundo factor, los indicadores claves radican, primordialmente, en el precio de las terminales; y en el tercero, el nivel de cumplimiento de los requisitos de las cargas y la composición de la cadena de suministro constituyen los ratios prioritarios.

Los nuevos paradigmas de la economía marítimo-portuaria ilustran la relevancia de las economías de escala y de aglomeración para la obtención de mejores resultados financieros, operaciones y logísticos. En esta línea se inscriben los procesos de concentración empresarial y de alianzas marítimas, derivados de las fusiones y adquisiciones de empresas, la posterior reducción de los players en los tableros económicos; y la reducción del poder de negociación de las Autoridades Portuarias en los mercados globales; aspectos que implican que estas enfaticen en la definición de su posicionamiento y de sus estrategias de marketing (González-Laxe, 2010).

Siep (2010) enfatiza sobre la diferenciación como ejes de la competitividad portuaria. Esto es, los puertos necesitan adoptar criterios de estrategia competitiva que incluyan su posicionamiento de cara a poder competir en las cadenas de suministro global. La selección de dichas estrategias de marketing resulta esencial y está muy determinada de cara a satisfacer a los clientes. Sigue el modelo de M. Porter, es decir, rivalidad entre los competidores, análisis de las amenazas de los nuevos competidores; examina el potencial de los sustitutos globales, evalúa los poderes de negociación del puerto; y toma nota de los poderes de negociación de los servicios portuarios.

Panayides y Song (2011) identifican un sistema de información y comunicación como una práctica necesaria de cara a incrementar los niveles de empleo, productividad y competitividad de los puertos. Dicho sistema de información y comunicación facilita la gestión eficiente de las operaciones de la cadena de suministro y permite alcanzar los objetivos programados.

Pardali y Kounoupas (2014) en el campo de la estrategias del marketing, especifican varios elementos básicos que son preciso tener en consideración. Los más significativos son: la promoción, la relación con la comunidad local, el desarrollo del comercio y la relación con los usuarios.

Calderinha (2014) distingue cinco grandes campos de análisis. Los relacionados con: a) la "position port"; es decir, los vinculados con la posición geográfica del puerto y de la terminal; b) factores "hard port"; aquellos relacionados con los aspectos que caracterizan físicamente el puerto; c) los factores "soft port"; es decir, los relativos a los aspectos no físicos que definen la gestión y el producto (como los 
servicios del puerto, la gobernanza, las tasas, los niveles de especialización, la calidad y la integración terrestre); d) los factores "hard terminal"; que enfatizan en las características físicas de las terminales, tales como equipamientos y muelles; y e) factores "soft terminal"; que inciden en los factores no físicos de los servicios de las terminales, esto es, en los modelos de gestión, la calidad, la fiabilidad, la imagen del terminal y los sistemas de información.

\section{Marketing portuario y escenarios estratégicos a largo plazo}

El mayor reto en la utilización del marketing consiste en atraer más clientes al mercado. Por tanto, las estrategias para captar más usuarios, subrayan dos aspectos: el primero, hace referencia a la necesidad de aumentar la cuota de participación en un mercado abierto y competitivo (estrategias de volumen); y, el segundo, en atraer nuevos clientes; y, sobre ellos, obtener el mayor potencial de crecimiento de los beneficios (estrategias de conectividad).

El contenido de las acciones de marketing, en cualquier caso, ha de estar en consonancia y ser adecuado a la rentabilidad de la empresa o de la actividad. En consecuencia, las tácticas para hacer crecer la rentabilidad, han de dirigirse hacia el aumento de la demanda de mercado, la cuota de participación de las empresas, los ingresos por clientes y, finalmente, la reducción de los costes variables por cliente o los propios gastos derivados de las acciones de marketing.

La contribución del marketing al funcionamiento de la actividad de una empresa está relacionada con las estrategias para aumentar la demanda de mercado, con las opciones para entrar o abandonar las posiciones comerciales y con las iniciativas tomadas para incrementar la cuota de participación. Pero, a su vez, está condicionada, por la necesidad de aumentar las ventas del producto y los cambios en sus costes variables. Finalmente, posee una relación inversa con las estrategias para mejorar la eficiencia en el uso de los recursos del marketing.

El mercado portuario al ser muy amplio y heterogéneo muestra varias notas diferenciadoras. En primer término, existen puertos muy innovadores y puertos líderes frente a un mercado que requiere de soluciones integradas. Pero el ritmo de crecimiento de los puertos es muy dispar, ya que depende de las características de los clientes, del posicionamiento de los productos y servicios que ofrece, de su adscripción a las cercanías de las rutas, y de otras influencias. Por ello, la especificidad portuaria subraya las ventajas relativas para entrar en el mercado: los precios relativos; la disponibilidad de servicios ofertados, los servicios integrados; y los aspectos relacionados con la reducción de riesgos y la seguridad.

Una forma de explicitar el mapa estratégico portuario es aquel que recoge un conjunto de objetivos que van respondiendo, simultáneamente, a una perspectiva económica, a los clientes, a los procesos y a los recursos. En tanto que las líneas estratégicas combinan el valor, la eficiencia, la integración y la rentabilidad. De esta forma, un puerto combina dos funciones, la primera, define instrumentos para facilitar el comercio y, la segunda, facilita la inserción a los demás sistemas modales y la integración sectorial. Por eso, los puertos procuran atender a tres objetivos básicos:

- alcanzar una mayor competitividad;

- mostrar altos niveles de calidad; y,

- generar el valor añadido más elevado posible de los servicios prestados.

En el primer caso, la mejora de la competitividad se logra por medio de actuaciones referidas a la existencia de infraestructuras y dotaciones de equipamientos básicos y especializados; a través de la leal competencia y transparencia en el mercado de los servicios portuarios evitando las distorsiones a la mencionada concurrencia; por medio de las tarifas y costes en los servicios portuarios que inciden en los costes operativos y en los tiempos de los servicios portuarios prestados; y por la eficiencia de los recursos humanos.

En el apartado de los rasgos definitorios de la calidad, los elementos claves son la tecnificación de los servicios; la capacitación profesional de los trabajadores; la disponibilidad de estándares de calidad; la seguridad y la confiabilidad de los sistemas logísticos portuarios; la protección del medio ambiente; y mantener la paz laboral.

Por último, en lo que hace referencia a la generación de valor añadido, los rasgos a mejorar se refieren a los desarrollos de las actividades de valor añadido en los servicios portuarios; a la obtención de ambiente propicio para la inversión; a la promoción de instalaciones portuarias de alto valor añadido; y a la automatización de los procesos.

En consecuencia, el planeamiento estratégico de un puerto toma nota de la metodología necesaria para estudiar las perspectivas económicas de la inversión efectuada y de los recursos necesarios para desarrollar, en condiciones competitivas, uno o varios negocios portuarios, teniendo en consideración los escenarios estratégicos a largo plazo, ya sean ofensivos o defensivos (figura 1).

\section{Marketing y estrategias en las orientaciones de mercado de la industria portuaria}

Los puertos en los últimos años han experimentado grandes cambios en lo que respecta a su organización. Al mismo tiempo han modificado sus relaciones tanto en lo que hace referencia al hinterland como en lo tocante a las relaciones con los usuarios. La mayor internacionalización del comercio y el nuevo posicionamiento de las compañías marítimas estipularon la presencia de los grandes operadores logísticos, capaces de una rápida adecuación a la hora de responder a las necesidades y de poder otorgar satisfacción a los clientes. Así las cosas, los mercados se han transformado; y la consecuencia de dichos cambios, es la mayor rivalidad y competencia que exigen nuevas infraestructuras y facilidades para operar en cada puerto (Cahoon y Notteboom, 2008).

Los puertos tradicionales fueron modificando sus hábitos y sus comportamientos; y, se afanaron en potenciar su promoción (UNCTAD, 1992). Las Autoridades Portuarias asumieron la obligación de promover una imagen positiva de sus actividades, cara a retener a clientes directos. En este sentido, 
Figura 1. Escenarios estratégicos a largo plazo para los puertos

\section{PLANES ESTRATÉGICOS OFENSIVOS}

\begin{tabular}{|c|c|c|}
\hline ESTRATEGIA BÁSICA I & ESTRATEGIA BÁSICA II & ESTRATEGIA BÁSICA III \\
\hline $\begin{array}{l}\text { Invertir para aumentar } \\
\text { las ventas y los servicios } \\
\text { a mercados existentes }\end{array}$ & $\begin{array}{l}\text { Invertir para mejorar la } \\
\text { posición competitiva }\end{array}$ & $\begin{array}{l}\text { Invertir para entrar en } \\
\text { nuevos mercados }\end{array}$ \\
\hline$\downarrow$ & $\downarrow$ & $\downarrow$ \\
\hline $\begin{array}{l}\text { Crecer en mercados } \\
\text { existentes }\end{array}$ & Mejorar los márgenes & $\begin{array}{l}\text { Diversificar el } \\
\text { crecimiento }\end{array}$ \\
\hline$\downarrow$ & $\downarrow$ & $\downarrow$ \\
\hline $\begin{array}{l}\text { - Crecimiento de la cuota } \\
\text { de mercado } \\
\text { - Crecimiento de los } \\
\text { ingresos por cliente } \\
\text { - Entrada en nuevos } \\
\text { segmentos de mercado } \\
\text { - Aumento de la demanda } \\
\text { del mercado }\end{array}$ & $\begin{array}{l}\text { - Mejora de la lealtad y } \\
\text { niveles de compras de los } \\
\text { clientes } \\
\text { - Mejora de las ventajas de } \\
\text { la diferenciación } \\
\text { - Disminución de los costes } \\
\text { de marketing }\end{array}$ & $\begin{array}{l}\text { - Entrada en mercados } \\
\text { nuevos relacionados y no } \\
\text { relacionados } \\
\text { - Entrada en nuevos } \\
\text { mercados emergentes } \\
\text { - Desarrollo de nuevos } \\
\text { mercados }\end{array}$ \\
\hline
\end{tabular}

\section{PLANES ESTRATÉGICOS DEFENSIVOS}

\begin{tabular}{|c|c|c|}
\hline ESTRATEGIA BÁSICA I & ESTRATEGIA BÁSICA II & ESTRATEGIA BÁSICA III \\
\hline Proteger la posición & Optimizar la posición & Desinvertir \\
\hline$\downarrow$ & $\downarrow$ & $\downarrow$ \\
\hline Mantener los beneficios & Maximizar los beneficios & Obtener cash-flow \\
\hline$\downarrow$ & $\downarrow$ & $\downarrow$ \\
\hline $\begin{array}{l}\text { - Proteger las cuotas de } \\
\text { mercado } \\
\text { - Desarrollar la lealtad del } \\
\text { cliente }\end{array}$ & $\begin{array}{l}\text { - Maximizar la contribución } \\
\text { neta del marketing } \\
\text { - Centrar el enfoque }\end{array}$ & $\begin{array}{l}\text { - Gestionar para obtener } \\
\text { tesorería } \\
\text { - Desinvertir y obtener } \\
\text { tesorería }\end{array}$ \\
\hline
\end{tabular}

Fuente: elaboración propia

cobran interés las estrategias de marketing que posibiliten el desarrollo comercial de los entornos portuarios.

No obstante, no son muy numerosos los trabajos que hacen referencia al marketing portuario. En las seminales aportaciones de la UNCTAD (1992) se hacía mención a las oportunidades de las acciones y a los conceptos de marketing aplicados a la organización portuaria bajo el nuevo rol que desempeñan los puertos al mudar su tradicional concepción de punto de transferencia modal y ser considerado, en la actualidad, como un centro de servicios. Posteriormente, la UNCTAD (1995) enfatiza sobre el marketing promocional. En la medida en que son muchas las variables que inciden de manera directa, en la relación o en el contacto con los clientes, las actuaciones de las Autoridades Portuarias en esta materia son muy recientes. Se definen cinco grandes apartados: en primer lugar, aumentar el conocimiento y la notoriedad del puerto; en segundo lugar, posibilitar el camino para viabilizar las ventas; en tercer lugar, mejorar la imagen del puerto; en cuarto lugar, aumentar la reputación del puerto; y, finalmente, destacar las cualidades de eficiencia a nivel de infraestructura y superestructura.

A medida que se incrementa el comercio marítimo y se afianzan los desarrollos portuarios, emergen nuevos desafíos para las Autoridades Portuarias. Por un lado, asistimos a una amplia diversificación de los modelos de gobernanza, con una gran complejidad de responsabilidades y estrategias en función de las apuestas de cada país o región econó- mica. De otra parte, también se asiste a un cambio notable en los objetivos finales de las Autoridades Portuarias, al mudar sus funciones tradicionales por unas nuevas, cifradas en la obtención de beneficios, lograr mayores niveles de conectividad, y disponer de una mayor autonomía de gestión. El nuevo contexto socio-económico contemporáneo también introduce nuevos parámetros en la realidad portuaria: una mayor transparencia y accountability; una mayor competencia inter-puertos; y un creciente peso de la iniciativa privada en la operativa marítimo-portuaria.

Las Autoridades Portuarias desarrollan, pues, dos grandes funciones (González-Laxe, 2010). La primera es facilitar la estructuración de los negocios; buscando una mayor competitividad, presentando altos niveles de calidad y tratando de maximizar el valor agregado de los servicios ofertados; y, la segunda trata de facilitar la integración sectorial y la inserción con otros modos de transporte del sistema. Bajo esta perspectiva se contemplan las dimensiones funcionales de las Autoridades Portuarias (tabla 1).

Tabla 1. Dimensiones estratégicas de las Autoridades Portuarias

\begin{tabular}{ll} 
Dimensión & Variables \\
\hline Económica & - Generar beneficio \\
& - Agregar valor para el cliente y para la \\
& sociedad. \\
\hline Clientes & - Ofertar líneas regulares \\
& - Poseer capacidad operacional \\
& - Mostrar tarifas competitivas. \\
\hline Procesos & - Productividad de instalaciones y \\
& operaciones \\
& - Integración real con la comunidad local \\
& - Ratio de gestión de costes y de \\
& inversiones \\
& - Promoción del puerto a nivel \\
& internacional. \\
& - Adecuación de infraestructuras físicas \\
& - Innovación tecnológica y equipos \\
Recursos & adecuados.
\end{tabular}

Fuente: elaboración propia

Ante estas expectativas, las Autoridades Portuarias pueden optar por expandir las tareas de difusión hacia distintas localizaciones: existen puertos que apuestan por el foreland, esto es, están volcados en la realización de inversiones en otras radas fuera del país; en segundo lugar, existen puertos que centran las acciones en torno al hinterland, es decir, actuaciones en el propio país; $y$, finalmente, existen puertos que enfatizan el planteamiento de estrategias basadas en el emprendimiento multinacional, o sea, en establecer convenios y acuerdos con otros puertos o empresas (Dooms, Van der Lugth y Parola, 2012).

Partiendo de las primeras concepciones sobre marketing portuario, la presencia de los puertos como agentes activos en el juego global, es decir, su respuesta a todas estas nuevas formas de liberalización y comercio globalizado, ha permitido afrontar el análisis de las estrategias de marketing y de comunicación en base a nuevos enfoques (tabla 2):

- la emergencia de las cadenas de suministro basadas en una fuerte contenerización de sus unidades de produc- 
Tabla 2. Clasificación de los distintos enfoques del marketing portuario

\begin{tabular}{|c|c|c|}
\hline Concepción & Enfoques & Autores \\
\hline \multirow{6}{*}{$\begin{array}{l}\text { Primeras concepciones orientadas } \\
\text { hacia los aspectos relacionados con la } \\
\text { competitividad y los servicios }\end{array}$} & - Marketing clásico y promocional & UNCTAD $(1992,1995)$ \\
\hline & - Competitividad & Stutchey (1991) \\
\hline & - Demanda, entorno y orientación & Schulten (1991) \\
\hline & $\begin{array}{l}\text { - Precios de los servicios portuarios } \\
\text { (tarificación, etc.) }\end{array}$ & Mester (1991) \\
\hline & - Nichos de mercado & Muhler (1991) \\
\hline & - Orientación organizativa & Stutchey (1991) \\
\hline \multirow{5}{*}{$\begin{array}{l}\text { Concepción del marketing orientado } \\
\text { hacia las terminales de contenedores } \\
\text { (Pardali y Kounoupas 2007, 2014) }\end{array}$} & - Desde la perspectiva de los shippers & Marti (1986) \\
\hline & $\begin{array}{l}\text { - Desde la perspectiva del hinterland y } \\
\text { de las infraestructuras }\end{array}$ & Cuadrado et al. (2004) \\
\hline & $\begin{array}{l}\text { - Desde la perspectiva de la eficiencia } \\
\text { técnica }\end{array}$ & Dong-Wook y Panayides (2007) \\
\hline & $\begin{array}{l}\text { - Desde la perspectiva de los hubs y de } \\
\text { las shipping lines }\end{array}$ & Meersman et al. (1999) \\
\hline & $\begin{array}{l}\text { - Desde la perspectiva del desarrollo } \\
\text { portuario }\end{array}$ & Baird (1999) \\
\hline \multirow{6}{*}{$\begin{array}{l}\text { Concepción orientada hacia el nuevo rol } \\
\text { del puerto }\end{array}$} & $\begin{array}{l}\text { - Referenciando las competencias y la } \\
\text { rivalidad externa }\end{array}$ & $\begin{array}{l}\text { Pardali y Stounoupoulos (2005), Heaver } \\
\text { et al. (2000), Lamonarca y Papa (2005) }\end{array}$ \\
\hline & $\begin{array}{l}\text { - Relacionado con la competitividad y la } \\
\text { integración vertical / horizontal }\end{array}$ & Verhoeff (1981), Song y Lee (2012) \\
\hline & $\begin{array}{l}\text { - Enfatizando en la inserción de las } \\
\text { cadenas de suministro }\end{array}$ & $\begin{array}{l}\text { Winkelmans (2003), Chlomuidis y Pallis } \\
\text { (1999), Parola y Musso (2007), Pallis y De } \\
\text { Langen (2006), Notteboom y Wilkenmans } \\
\text { (2002), Paixao y Marlow (2003), Song } \\
\text { (2003), Chlomoudis et al. (2003), Cahoon } \\
\text { y Hecker (2005), Cahoon y Notteboom } \\
\text { (2008) }\end{array}$ \\
\hline & - Función de los usuarios & $\begin{array}{l}\text { Martin y Thomas (2001), Suyyckens } \\
\text { (1985), Notteboom (2002), Notteboom y } \\
\text { Wilkenmans (2002) }\end{array}$ \\
\hline & - El puerto como producto & Pardali (2007), Parola et al. (2016) \\
\hline & - Vínculo foreland-hinterland & $\begin{array}{l}\text { Pardali y Stathoupoulos (2005); Pais et } \\
\text { al. (2012) }\end{array}$ \\
\hline \multirow{4}{*}{$\begin{array}{l}\text { Concepción basada en la imagen, } \\
\text { percepción y satisfacción de los clientes }\end{array}$} & - Comunicación & Siep (2010) \\
\hline & - Reputación & $\begin{array}{l}\text { Juang y Roe (2009), Onut,et al. (2011), } \\
\text { Chang y Lee (2008), Cheon (2007) }\end{array}$ \\
\hline & - Satisfacción de los clientes & $\begin{array}{l}\text { Liu et al. (2009), Caldeirinha et al. } \\
\text { (2013) }\end{array}$ \\
\hline & - Agilidad ante los cambios & Woo et al. (2011), Liu et al. (2009) \\
\hline
\end{tabular}

Fuente: elaboración propia

ción, posibilitó formas específicas de difusión de las capacidades portuarias que, aún hoy, se revelan como altamente correlacionadas con el desarrollo económico de la región portuaria de influencia (Pardali y Kounoupas, 2007, 2014);

- factores externos y ajenos que actúan sobre los puertos, modificando su organización y redefiniendo su rol: gestión de la rivalidad (puertos directamente competidores), integración vertical/horizontal, inserción en las cadenas de suministro, roles de usuario, el puerto como producto y la explotación comercial del vínculo entre hinterland y foreland.
- finalmente, una tercera diferenciación, más novedosa temporalmente, está orientada exclusivamente en términos de la percepción que tienen los principales clientes: navieros, cargadores y consignatarias. En este sentido, Siep (2010) realiza una distinción entre objetivos básicos del marketing portuario $y$, entre los más significativos se pueden citar la atracción de nuevos clientes y la información a clientes actuales, la información dirigida hacia la comunidad local, la información compartida con el sector marítimo y, por último, el panorama de servicios del puerto y su imagen de marca. 


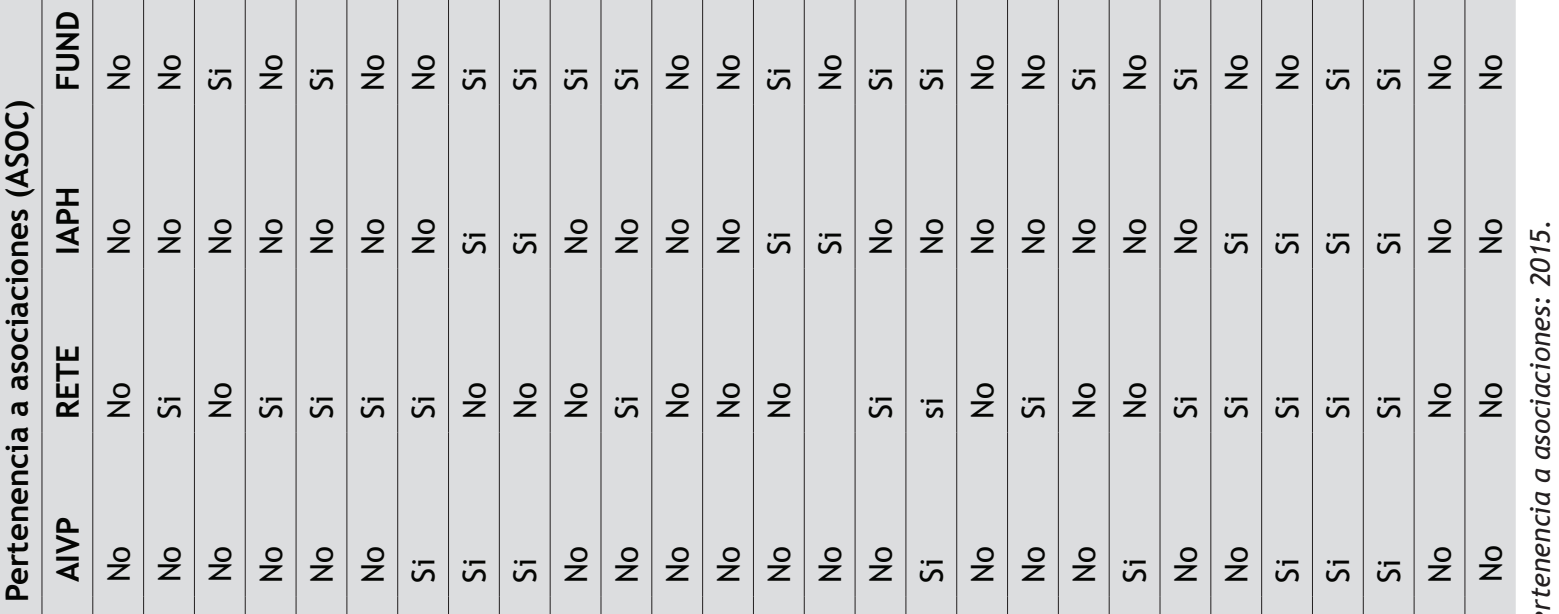

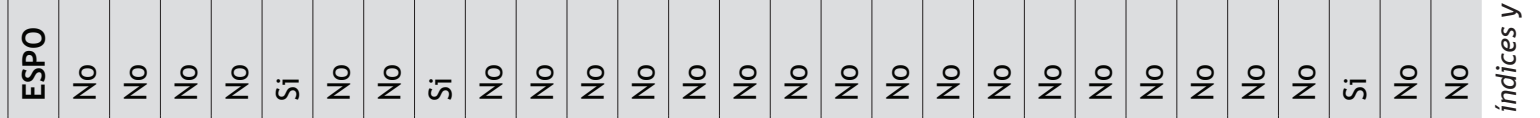

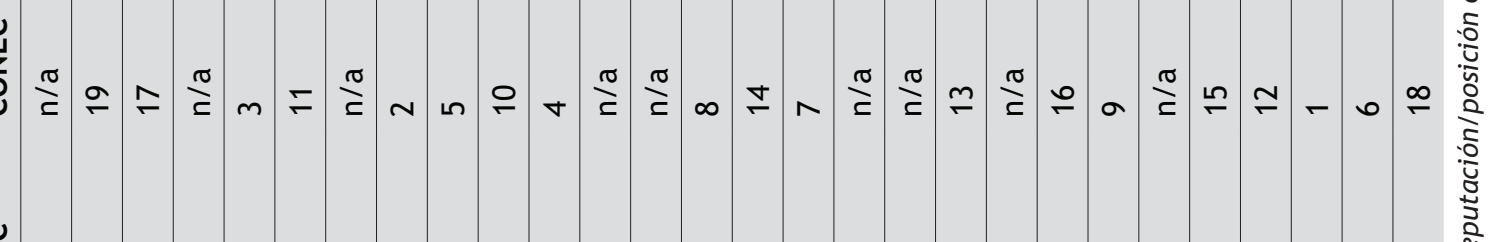

U⿺辶一

$\stackrel{0}{\underline{0}}$

:

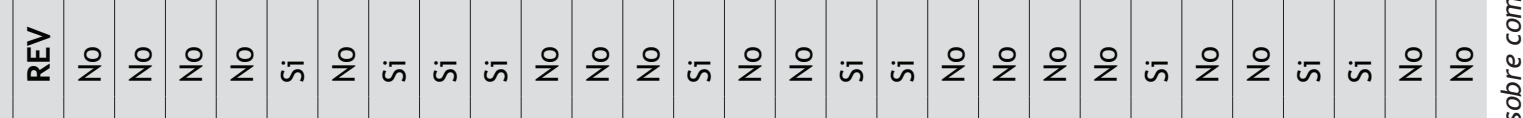

.5

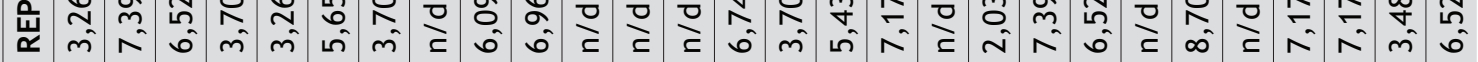
ô

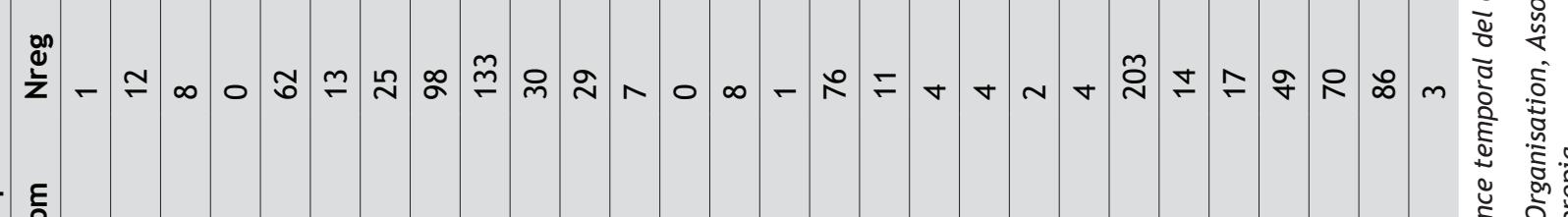

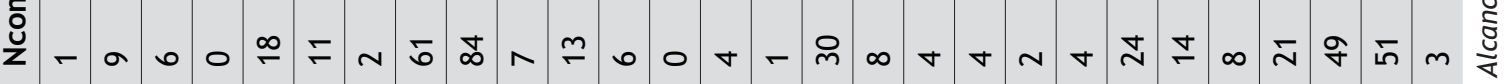

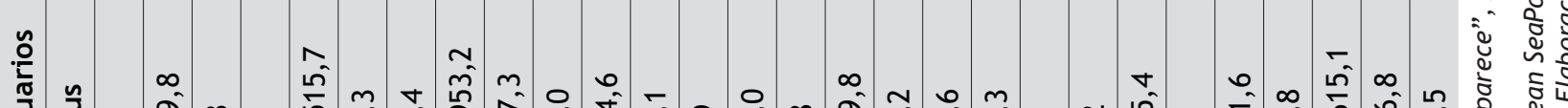

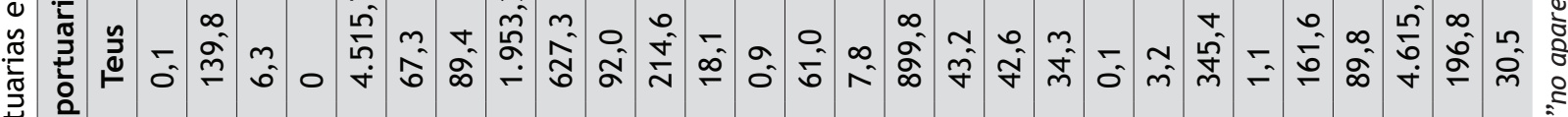
约

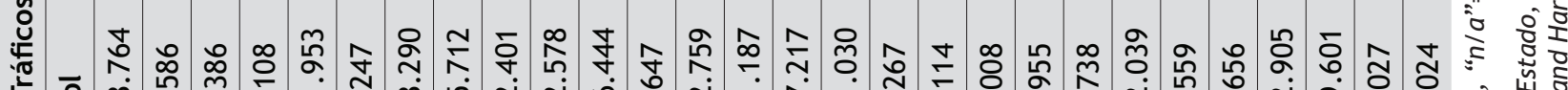

훈

लं

$\frac{\pi}{\circ}$

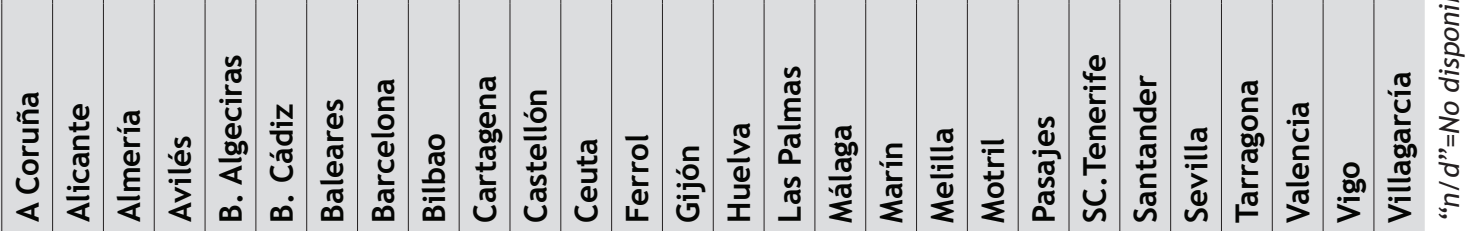

잉 
Tabla 3b. Autoridades Portuarias españolas: indicadores de promoción y de desarrollo de acciones de marketing (leyenda)

VOL Volumen total de tráficos de graneles líquidos, sólidos y mercancía general (expresados en miles de Tns.) (2015)

TEUS Volumen tráficos correspondientes a mercancías contenerizadas (expresados en miles de TEUS) (2015)

Ncom Número de compañías marítimas que utilizan el puerto

Nreg Número de servicios de línea regular en el puerto

REPint Posición del concepto "reputación" ("fiabilidad de los servicios portuarios"+"localización portuaria"+"reputación internacional") sobre objetivos declarados del puerto (0="prioridad nula"; 5="prioridad media"; 10="máxima prioridad") (González et al., 2015)

REV Publicación de anuncios en revistas especializadas (Containeration International $(\mathrm{Cl})$; International Bulk Journal (IBJ); Passenger Ship Technology (PST)

ACC Posición en el ranking español según el índice de accesibilidad portuaria (IAP). Incluye condiciones técnicas del puerto y movimientos de buques; capacidad y servicios del puerto junto a ratios de conectividad; asimismo se incluyen ratios económicos y financieros. Se efectúa para todas las Autoridades Portuarias españolas (González-Laxe , 2011)

CONEC Posición en el ranking español según índice de conectividad portuaria (ICP), reflejado para los puertos que movilizan contenedores; y elaborado por Valencia Port (2016). Incluye la suma de navieras que ofrecen servicios en cada puerto junto al número de líneas, la capacidad, el número de buques y su tamaño, así como la frecuencia de los servicios ofertados y los puertos de destino.

ESPO Pertenencia a la European SeaPorts Organisation (formando parte del Consejo Ejecutivo)

AIVP Pertenencia a Association Internationale Villes et Ports (solo se consideran socios)

RETE Pertenencia a la Association for the Cooperation between Ports and Cities (RETE) (se considera tanto

ASOC socios como colaboradores)

IAPH Pertenencia a International Association of Ports and Harbours (se consideran adherentes).

FUND ¿Posee la Autoridad Portuaria algún Organismo/Agrupación/ Fundación/Instituto para la promoción o prestación servicios propios?

Fuente: elaboración propia

\section{El caso español}

El sistema portuario español está compuesto por 46 puertos agrupados en 28 Autoridades Portuarias (tablas 3a y $3 \mathrm{~b})$. Se caracteriza por ser un conjunto muy heterogéneo, con características muy dispares entre unos y otros en lo tocante a su tamaño, a los volúmenes de cargas y a sus niveles de especialización. También son ostensibles las diferencias entre sus conectividades y accesibilidades portuarias y su presencia en las diferentes fachadas marítimas (atlántica, mediterránea o insular). Igualmente, son notables las desiguales características en lo que respecta a los flujos de mercancías que movilizan, ya sean nacionales, tráficos import/export o tráficos de transhipment.

No hay duda de que la capacidad de captación de tráficos en los puertos españoles está condicionada tanto por sus políticas de atracción, (y con ello se deben anotar la amplia panoplia de ventajas determinadas por la selección portuaria, ya sea desde el punto de vista técnico, infraestructural y de disponibilidades, como desde las perspectiva económica y comercial); como también, en segundo lugar, por el desarrollo de aquellas acciones que tratan de mejorar y contribuir al posicionamiento internacional y logístico.
Los recientes trabajos sobre el tema de la Port Choice así lo avalan y demuestran. Por eso, a la hora de poder medir cada uno de los índices que posee cada Autoridad Portuaria española se ha procedido a establecer cinco indicadores que nos permitan auscultar el grado y el nivel de esfuerzo desplegado en la promoción y en las labores de marketing. Se han seleccionado como elementos claves: el volumen de tráfico portuario; la presencia de compañías marítimas y de servicios regulares que operan en el puerto; el análisis de la reputación del puerto; la posición en los índices de conectividad y accesibilidad, y; la presencia en asociaciones/agrupaciones sectoriales.

Para la obtención de los datos se han utilizado fundamentalmente fuentes primarias de información basadas en diferentes niveles de gobernanza portuaria (Puertos del Estado, European SeaPorts Organisation, Association Internationale Villes et Ports, Association for the Cooperation between Ports and Cities, International Association of Ports and Harbours).

Con los datos obtenidos de los índices de cada Autoridad Portuaria, que tratan de medir el grado y el nivel de esfuerzo desplegado en la promoción de las estrategias de marketing portuario, se ha aplicado un análisis de componentes prin- 
cipales (figura 2). La utilización de esta técnica como base metodológica para temas específicos de selección portuaria puede ser rastreada en (González-Laxe et al., 2015; Sayareh et al., 2014; Aronietis et al., 2010 y Chang et al., 2008). Los resultados obtenidos cumplen las requisitos estándar de bondad de ajuste para esta técnica multivariante (medida de Kaiser-Meyer-Olkin de 0,733 -calificable como notable-; y rechazo de la hipótesis de esfericidad de Bartlett -homogeneidad de varianzas- al 99\%).

Es preciso señalar como una limitación del estudio el hecho de que, a pesar del cumplimiento de los requisitos estadísticos en la aplicación de la técnica de segmentación, la precisión de la factorización propuesta debería ser reforzada por una posterior sub-muestra más amplia, añadiendo quizá como variables de input nuevas características que, en este caso, no ha sido posible registrar.

El procedimiento, no obstante, ha conseguido diferenciar de modo ortogonal dos pautas de comportamiento: una primera componente (F1) que enfatiza, de cara a los posibles clientes, la fortaleza del puerto en lo que respecta al número de compañías (Ncom), número de servicios regulares (Nreg), indicador de accesibilidad (ACC) y grado de pertenencia a asociaciones sectoriales (ASOC), en lo que se podría denominar una estrategia de marketing centrada en el aumento del volumen de mercancías estrategia de volumen; y, en segundo lugar, una segunda componente (F2), que se centra en la conectividad (CONEC) y en la reputación internacional (REPint) como elementos centrales en su estrategia de marketing, en lo que se podría denominar una estrategia de conectividad.
En base a esta descomposición factorial, las estrategias de marketing portuario de las Autoridades Portuarias españolas se diferencian en cuatro tipos, en función de la posición de cada puerto con respecto a los cuadrantes delimitados por el análisis de componentes principales:

- Los puertos situados en el primer cuadrante (F1>0, F2>0), contemplan estrategias de marketing tanto desde el punto de vista del volumen como de la conectividad. Se trata de Las Palmas, Tarragona, Valencia y Bilbao, que consiguen enfocar desde un punto de vista integral, la tarea de promoción de su oferta de servicios.

- Los puertos situados en el segundo cuadrante (F1>0, $\mathrm{F} 2<0$ ), priorizan las estrategias de conectividad sobre las estrategias de volumen. Se trata de puertos que no están tan centrados en conseguir, al menos a corto plazo, un aumento significativo en su volumen de mercancías, como de desplegar políticas de promoción comercial de su cartera de servicios: B. Cádiz, Málaga, Motril, Huelva, Santander, Gijón, Cartagena, Ceuta, Pasajes, Vilagarcía, Almería y Alicante.

- Los puertos situados en el cuarto cuadrante (F1<0, F2>0), priorizan las estrategias de volumen sobre las estrategias de conectividad. Algunos son puertos importantes en volumen de mercancías (B. Algeciras, Barcelona), mientras que otros se sitúan en un orden secundario con respecto a este parámetro (Vigo y S.C. Tenerife). Se trata de una situación en la cual se intenta mantener las inercias favorables ya existentes en lo que respecta a la cartera de clientes y a la mejora/mantenimiento de las infraes-

Figura 2. Análisis de componentes principales: Ncom, Nreg, REPint, ACC, ASOC (estrategias de volumen); CONEC, ASOC (estrategias de conectividad)

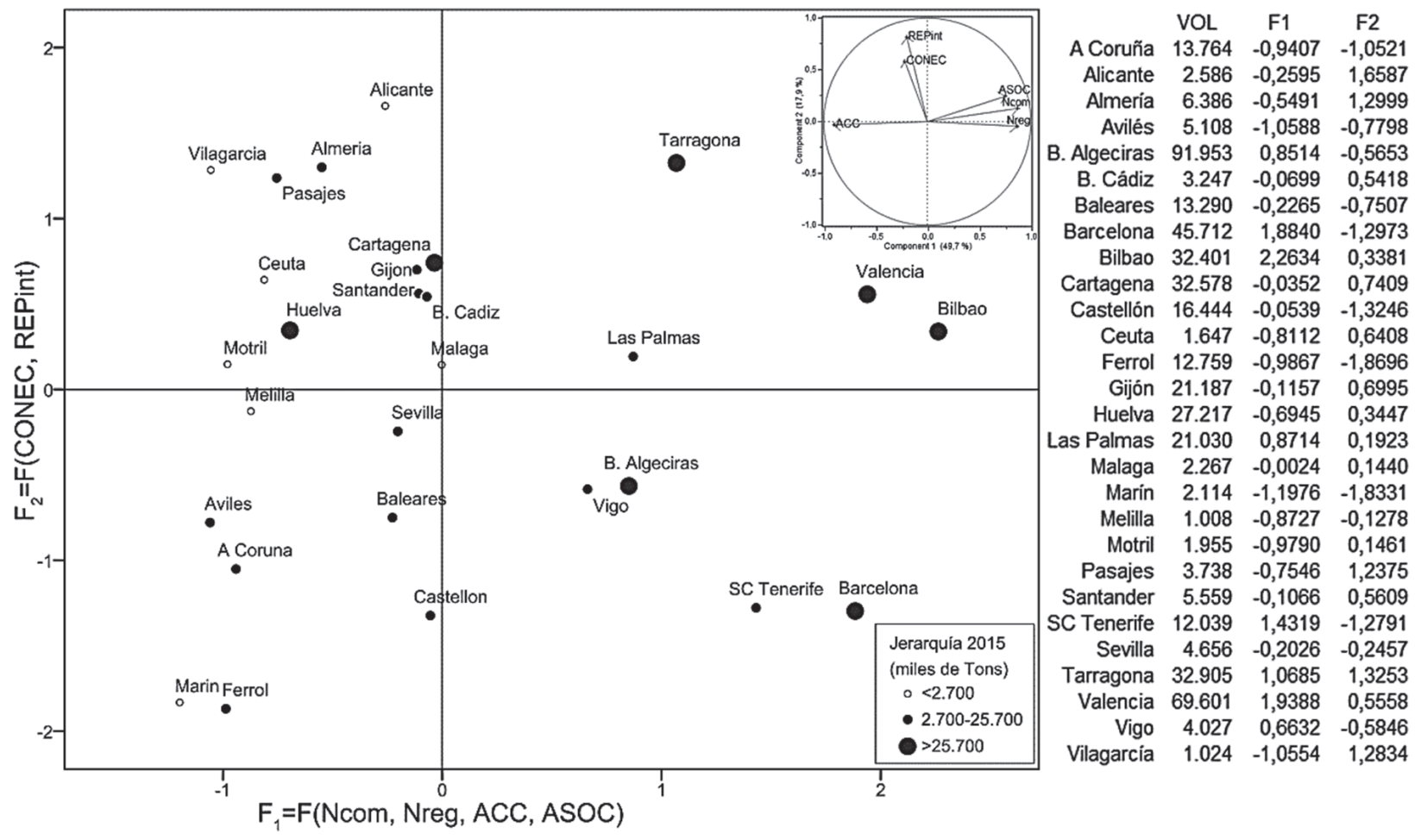

KMO: 0.733, Test de Bartlett: $a=0.001$. Fuente: elaboración propia 
tructuras existentes; pero quizá, en algún caso, se podría estar evidenciando una desconexión con respecto a la cadena global de suministros, en el sentido de desatender las políticas de exploración comercial de nuevos nichos de mercado (estrategias de conectividad).

- El último grupo $(\mathrm{F} 1<0, \mathrm{~F} 2<0)$ está compuesto por puertos que quizá no estén realizando un correcto desempeño de las tareas de marketing con respecto al promedio del sistema portuario español, mostrando un amplio margen de mejora tanto en las estrategias de conectividad como en las estrategias de volumen, lo que debería ser objeto de especial atención de cara al mantenimiento futuro de funciones portuarias no deficitarias.

\section{Conclusiones}

En primer lugar, cabe señalar que los puertos tradicionales modificaron sus hábitos y sus comportamientos y, actualmente, tratan de potenciar su promoción en un mercado global donde existe la mayor rivalidad y competencia, y donde cada vez se exigen nuevas infraestructuras y mayores facilidades para operar.

Las primeras tentativas enunciadas hace tres décadas, para extender el análisis del marketing al territorio económico específico de la actividad portuaria, estaban centradas en los ámbitos convencionales donde se ejercen las acciones de difusión comercial: aumento del conocimiento y notoriedad del puerto, viabilidad de las ventas, mejora de la imagen, aumento de la reputación, y difusión de las mejores cualidades en términos e infra y super-estructura.

Pero el desarrollo del comercio globalizado, así como la emergencia y consolidación de nuevos ámbitos territoriales para los puertos (foreland, hinterland y espacios de hibridación entre estos dos conceptos) ha posibilitado la expansión de la actividad tradicional de marketing portuario hacia tres nuevos enfoques complementarios: en primer lugar, las valiosas lecciones aprendidas en base a la expansión del tráfico marítimo contenerizado y de su impacto en los puertos de todo el mundo, lo que ha supuesto, a su vez, nuevas ventanas de oportunidad para la promoción portuaria en temas tecnológicos como las nuevas formas de trazabilidad, los nuevos y crecientes tamaños de buque, el transporte contenerizado de materias primas, alimentos y materiales peligrosos, o los nuevos modos logísticos just-in-time y door-to-door; en segundo lugar, el cambio en el rol de los puertos tanto como entidades que vivían de espalda a las ciudades donde estaban situados (el concepto de port-city), como en su nueva función de organismos mediadores de una gran parte de la actividad de importación/exportación que ha posibilitado que las estrategias de marketing puedan dirigirse hacia los canales competidores, y a la totalidad de la cadena de suministro (foreland+hinterland); en tercer lugar, la importancia que la imagen corporativa adquiere en las ideas de negocio actuales, que también se extiende a la actividad de marketing portuario, posibilitando el establecimiento de una nueva relación de proximidad con los clientes basada en el diseño de políticas innovadoras de comunicación, de mejora de la reputación internacional, de la satisfacción de los clientes, y de la respuesta ágil ante los cambios.
Todo este panorama, que podríamos determinar definitorio de una "nueva generación de gobernanza portuaria", ha sido impulsado de manera muy nítida en las dos últimas décadas aprovechando la globalización de la cadena de suministro y la inserción del transporte marítimo como elemento fundamental de esta dinámica. Pero, de igual manera, en la actual fase recesiva, también se asiste a una consolidación de determinados enclaves portuarios regionales aprovechando las reducciones de la actividad en el transporte, las nuevas reubicaciones geográficas de las unidades de producción, y los mayores niveles de decisión con que los agentes privados, en el seno de las Autoridades Portuarias, buscan las cargas de mercancías y ofertan los servicios.

En el caso particular de los Puertos de Interés General de España, las estrategias actuales de marketing portuario parecen estar orientadas hacia dos grandes líneas generales: las estrategias de volumen, que tratan de priorizar el mantenimiento de las dinámicas positivas ya existentes en términos de número de compañías que operan en el puerto, número de servicios regulares, parámetros de accesibilidad y pertenencia a asociaciones sectoriales; y las estrategias de conectividad que no se centran tanto en el aspecto cuantitativo de la actividad portuaria como en el cualitativo, en el sentido de priorizar operaciones de marketing que prioricen la mejora de la reputación internacional y el aumento de los parámetros de conectividad.

La situación ideal es que los equipos de promoción comercial de las Autoridades Portuarias prioricen un mix de las dos estrategias, a fin de cubrir todas las posibilidades anteriormente enunciadas, pero en la realidad, existen puertos que han optado (queda por decidir si correctamente o no) por darle más énfasis a la alternativa de volumen o a la de conectividad, y también un grupo de puertos que muestran indicadores excesivamente reducidos en el desempeño de ambas estrategias de marketing portuario, lo que debería suponer un elemento de reflexión adicional, tanto para cada una de las Autoridades Portuarias individuales, como para el Organismo Público Puertos del Estado, como entidad coordinadora y reguladora del sistema estatal de Puertos de Interés General. 


\section{Bibliografía}

Anholt, S., 2002. Foreword. Journal of Brand Management, 9, 229-239.

Aronietis, R., Van de Voorde, E., and Vanelslander, T., 2010. Port competitiveness determinants of selected European ports in the containerized cargo market. University of Antwerp.

Baird, A.J., 1999. Container Vessels in the New Millennium: Implications for Seaports. 1999 IAME Conference. Halifax, USA.

Branch, A., 1998. Maritime Economics. Management \& Marketing. London: Cheltenham

Cahoon, S., 2007. Marketing Communications Strategies for Seaports: a matter of survival and growth. Maritime Policy and Management, 34(2), 151-168. doi: 10.1080/03088830701240342

Cahoon, S., and Hecker, R., 2005. Seaports in the New Economy: Using Services Marketing Strategies to Increase Cargo Throughput in a Hypercompetitive Environment. 2005 IAME Conference. Limassol, Cyprus.

Cahoon, S., and Notteboom, T., 2008. Port marketing tools in a logistics-restructured market environment: the quest for port loyalty. 2008 IAME Conference, Dalian, China.

Caldeirinha, V., Felicio, A., and Dionisio, A., 2013. The container terminal characteristics and customer's satisfaction (CEFAGE-UE, Working Paper 2013/14). Recuperado de http://www.cefage.uevora.pt/en/content/ download/3867/48563/version/1/file/2013_14.pdf

Caldeirinha,V., 2014. Influencia das características do porto/terminal de contentores no seu desempenho. Tesis, (Dr). Universidade de Evora. Recuperado de http://dspace.uevora.pt/rdpc/handle/10174/12237

Caldwell, N., and Freire, J.R., 2004. The differences between branding a Country, a Region, and a City: Applying the Brand Box Model. Journal of Brand Management, 12(1), 50-61. doi: 10.1057/palgrave.bm.2540201

Chang, Y., and Lee, S., 2008. Port selection factors by shipping lines: different perspectives between trunk liners and feeder's services providers. Journal of Marine Policy, 32(6), 877-885. doi: 10.1016/j.marpol.2008.01.003

Cheon, S., 2007. Evaluating impacts of institutional reforms on port efficiency changes: malmquist productivity index for models container ports. Berkeley: University of California.

Chlomoudis, C. I., Karalis, V. A., and Pallis, A. A., 2003. Port reorganisation and the worlds of production theory. European Journal of Transport and Infrastructure Research, 3(1), 77-94.

Chlomoudis, C.I., and Pallis, A.A., 1999. The need for a New Philosophy of Port Management and Organisation: Effective Responses to Contemporary Challenges. European Research Studies, 2(2), 91-103.

Cuadrado, M., Frasquet, M., and Cervera, A., 2004. Benchmarking the port services: a customer orientated proposal. Benchmarking, 11(13), 320-330. doi: 10.1108/14635770410538781

Dong-Wook, S, Panayides, P., 2007. Global supply chain and port/terminal: Integration and competitiveness. 2007 International Conference on Logistics, Shipping and Port Management. Taipei, Taiwan.
Dooms, M., Van der Lugth, L., and Parola, F., 2012. Current structure of research collaboration: from strategy to Taipei and beyond. 2012 IAME Conference, Taipei, Taiwan.

González-Laxe, F., 2010. Port marketing strategy and challenges of maritime globalization. En Coto, P. et al., eds. Essays on Port Economics. Berlin: Springer, 5-18.

González-Laxe, F., 2011. La Accesibilidad y conectividad portuaria. Boletín Económico de ICE, 3011, 35-46.

González-Laxe, F., Freire-Seoane, M.J., and Pais-Montes, C. 2015. Port policy and port choice: the spanish case. International Journal of Transport Economics, 42(4), 1-25

Heaver, T., Meersman, H., Moglia, F., and Van de Voorde, E., 2000. Do mergers and alliances influence European shipping and port competition?. Maritime Policy \& Management, 27(4), 363-373.

Juang, Y., and Roe, M., 2009. Study on success factors of development strategies for intermodal freight transports systems. $8^{\text {th }}$ International Conference of Eastern Asia Society or Transportation Studies, Surabaya, Indonesia.

Kotler, P., 2000. Marketing management. London: Prentice Hall International.

Kotler, P., and Getner, D., 2002. Country as a Brand, Product and Beyond: A place Marketing and Brand Management Perspective. Journal of Brand Management, 9(4), 249-261. doi: 10.1057/palgrave.bm.2540076

Lamonarca, M., and Papa, P., 2005. Port Governance: new Challenges for Port Authorities. 2005 IAME Conference. Limassol, Cyprus.

Liu, W., Xu, H., and Zhao, X., 2009. Agile service oriented shipping companies in the container terminal. Transport, 24(2), 143-153. doi: 10.3846/16484142.2009.24.143-153

Marti, B.E., 1986. Marketing Strategies: a Container Foreland Study of the Port of Miami. Geoforum, 17(3), 375382. doi: 10.1016/0016-7185(86)90005-9

Martin, J., \& Thomas, B. J., 2001. The container port community. Maritime Policy and Management, 8(3), 279292.

Meersman, H., Moglia, F., Van de Voorde, E., 1999. Mergers and Alliances in Liner Shipping: What do European port authorities have top fear? International Association of Maritime Economists. 1999 IAME Conference. Halifax, USA.

Mester, B., 1991. Marketing from the port's point of view. In Stutchey, R.W. (Ed.) Port Management Textbook (pp. 15-32. Bremen, Institute of Shipping and Logistics.

Muhler, G., 1991. Niche Marketing. In Stutchey, R.W. (Ed.) Port Management Textbook (pp. 15-32). Bremen, Institute of Shipping and Logistics.

Nandas, S., 2005. An exploration of the brand identity, brand image linkage: a communication perspective. Journal of Brand Management, 12(4), 254-279. doi: 10.1057/palgrave.bm.2540222

Notteboom, T., 2002. Consolidation and contestability in the European container handling industry. Maritime Policy and Management, 29(3), 257-269. doi: 10.1080/03088830210132614

Notteboom, T., 2011. Current Issues in shipping, ports and logistics. Antwerp: Vubpress 
Notteboom, T., and Winkelmans, W., 2001. Structural change in logistics: how will port face the challenge?. Maritime Policy and Management, 28(1), 71-89. doi: 10.1080/03088830119197

Notteboom, T., and Winkelmans, W., 2002. Stakeholder's relations management in ports: dealing with the interplay of forces among stakeholders in a changing competitive environment. 2002 IAME Conference, Panama City, Panama.

Onut, S., Tuzkanya, V., and Torum, E., 2011. Selectivity container port via a fuzzy ANEP-based approach. A case study on the Marmara region Turkey. Transport Policy, 18(1), 182-193. doi: 10.1016/j.tranpol.2010.08.001

Pais, C., Freire, M.J., González, F., 2012. General cargo and containership emergent routes: a complex networks description. Transport Policy, 24(2), 126-140. doi: 10.1016/j.tranpol.2012.06.022

Paixao, A., and Marlow, P.B., 2003. Fourth generations ports - a question of agility?, International Journal of Physical Distribution \& Logistics Management, 33(4), 355-376. doi: 10.1108/09600030310478810

Pallis, A. A., Vitsounis, T. K., De Langen, P. W., and Notteboom, T. E., 2011. Port economics, policy and management: Content classification and survey. Transport Reviews, 31(4), 445-471. doi: 10.1080/01441647.2010.530699

Pallis, A., and De Langen, P.W., 2006. Analysis of the benefits of intra-port competition. International Journal of Transport Economics, 33(1), 69-86.

Pando, J., Araujo, A., Maqueda, F., 2005. Marketing management at the world's major ports, Maritime Policy \& Management, 32(2), 67-87. doi: 10.1080/03088830500097414

Pardali, A., 2007. Port Economics and Policy. Atenas: Stamoulis

Pardali, A., and Kounoupas, E., 2007. The Application of Marketing Strategies in the Container Seaport Market. 5th International Congress on Maritime Technological Innovations and Research. Barcelona, España.

Pardali, A., and Kounoupas, E., 2014. Market orientations and business performance in the container port industry. Journal of Shipping and Ocean Engineering, 4(7-8), 173-184.

Pardali, A., and Stathopoulos, C., 2005. Port Competition: The case of the Greek Port Industry. 2005 IAME Conference. Limassol, Cyprus.

Parola, F., and Musso, E., 2007. Market structures and competitive strategies: the carrier-stevedore arm-wrestling in northern European ports. Maritime Policy and Management, 34(3), 259-278. doi: 10.1080/03088830701343369

Parola, F., Pallis, A.A., Risitano, M., and Ferreti, M., 2016. Marketing strategies of Port authorities: A multidimensional framework. 2016 EURAM Conference, Paris, Francia.

Plomaritou, E., 2006. Marketing of Shipping Companies. Atenas: Athens Stamoulis Publications.

Robinson, R., 2002. Ports as elements in value-driven chain systems: the new paradigm. Maritime Policy and Management, 29(3), 241-255. doi: 10.1080/03088830210132623

Sayareh, J., and Alizmini, H.R., 2014. A hybrid decision-making model for selecting container seaport in the Persian Gulf. The Asian Journal of Shipping and Logistics, 30(1), 75-95.
Schulten, U., 1991. Marketing from the Customer's point of view. En Stutchey, R.W., ed., Port Management Textbook. Bremen, Institute of Shipping and Logistics (1532).

Siep, J., 2010. Marketing communication strategies for seaports. Introduction to Maritime Economics and Logistics. Recuperado de bit.ly/2pujwXw

Song, D. W., and Lee, E. S., 2012. Coopetitive networks, knowledge acquisition and maritime logistics value. International Journal of Logistics Research and Applications, 15(1), 15-35. doi: 10.1080/13675567.2012.662949

Song, D.W., 2003. Port co-opetition in concept and practice. Maritime Policy \& Management, 30(1), 29-44. doi: 10.1080/0308883032000051612

Stutchey, R.W., 1991. Port Management Textbook: Port Marketing. Bremen: Institute of Shipping and Logistics

Suyckens, F., 1985. Administration and management of the port of Anwerp. Maritime Policy and Management, 12(3), 181-194.doi: 10.1080/03088838500000025

Tongzon, J.L., and Sawant, L., 2007. Port choice in a competitive environment: from the shipping lines' perspective. Applied Economics, 39(4), 477-492. doi: $10.1080 / 00036840500438871$

UNCTAD, 1992. Port marketing and the challenge of third generation port. Génève: UNCTAD

UNCTAD, 1995. Marketing promotion tool for port. Génève: UNCTAD

Valencia Port, 2016. Índice de Accesibilidad Portuaria. Recuperado de http://www.valenciaport.com/es/Paginas/default.aspx

Van der Lugth, L., and De Langen, P., 2007, Julio. Port Authority Strategy: beyond the landlord a conceptual approach. 2007 IAME Conference, Atenas, Grecia.

Verhoeff, J.M., 1981. Seaport Competition: Some fundamental and political aspects. Maritime Policy and Management, 8(2), 49-60. doi: 10.1080/03088838100000022

Winkelmans, W., 2003, Mayo. Port Competitiveness and Port Competition. Two of a kind?. 23rd IAPH World Ports Conference, Durban, South Africa.

Woo, S. H., Pettit, S., and Beresford, A. K., 2011. Port evolution and performance in changing logistics environments. Maritime Economics \& Logistics, 13(3), 250-277. doi: $10.1057 / \mathrm{mel} .2011 .12$ 